\title{
Optimal shapes of compact strings
}

\author{
Amos Maritan ${ }^{\ddagger}$, Cristian Micheletti ${ }^{\ddagger}$, Antonio Trovato $^{\ddagger} \&$ \\ JAYANTH R. BANAVAR ${ }^{\dagger}$
}

\begin{abstract}
$¥$ International School for Advanced Studies (S.I.S.S.A.), Via Beirut 2-4, 34014 Trieste, Istituto Nazionale di Fisica della Materia and the Abdus Salam International Center for Theoretical Physics, Trieste, Italy

$\dagger$ Department of Physics and Center for Materials Physics, 104 Davey Laboratory, The Pennsylvania State University, University Park, Pennsylvania 16802, USA
\end{abstract}

Optimal geometrical arrangements, such as the stacking of atoms, are of relevance in diverse disciplines [1, 2, 3, 4, 5]. A classic problem is the determination of the optimal arrangement of spheres in three dimensions in order to achieve the highest packing fraction; only recently has it been proved [1, 2] that the answer for infinite systems is a face-centred-cubic lattice. This simply stated problem has had a profound impact in many areas [3, 4, 5], ranging from the crystallization and melting of atomic systems, to optimal packing of objects and subdivision of space. Here we study an analogous problem-that of determining the optimal shapes of closely packed compact strings. This problem is a mathematical idealization of situations commonly encountered in biology, chemistry and physics, involving the optimal structure of folded polymeric chains. We find that, in cases where boundary effects [6] are not dominant, helices with a particular pitchradius ratio are selected. Interestingly, the same geometry is observed in helices in naturally-occurring proteins. 
The problem of placing spheres in three dimensions in order to attain the highest density was first posed by Kepler and has attracted much interest culminating in its recent rigorous mathematical solution [1]. The close-packed hard sphere problem may be restated in an alternative manner, more convenient for numerical implementation, as the determination of the arrangement of a set of points in a given volume that results in the minimum distance between any pair of points being as large as possible [6]. It is notable that the resulting 'bulk' optimal arrangement exhibits translational invariance in that, far from the boundaries, the local environment is the same for all points.

In this letter, we introduce a new problem pertaining to the optimal shapes of compact strings. Consider a string (an open curve) in three dimensions. We will utilize a geometric measure [7] of the curve, the 'rope-length', defined as the arc length measured in units of the thickness, which has proved to be valuable in applications of knot theory [7, 8, 9, 10, 11, 12]. The thickness $\Delta$ denotes the maximum radius of a uniform tube with the string passing through its axis, beyond which the tube either ceases to be smooth, owing to tight local bends, or it self-intersects. Our focus is on finding the optimal shape of a string of fixed arc length, subject to constraints of compactness, which would maximize its thickness, or equivalently minimize its rope length.

Following the approach of Gonzalez and Maddocks [10], who studied knotted strings, we define a global radius of curvature as follows. The global radius of curvature of the string at a given point is computed as the minimum radius of the circles going through that point and all other pairs of points of the string. It generalizes the concept of the local radius of curvature (the radius of the circle which locally best approximates the string) by taking into account both local (bending of the string) and non-local (proximity of another part of the string) effects. For discretized strings the local radius of curvature at a point is simply the radius of the circle going through the point and its two adjoining points. The minimum of all the global radii then defines the thickness, i.e. the minimum radius of the circles going through any triplet of discrete points. This coincides with the previous definition in the continuum limit, obtained on increasing the number of discretized points (assumed to be equally spaced) on the string keeping the string length fixed [10].

We used several different boundary conditions to enforce the confinement of the string. The simplest ones discussed here are the confinement of a string of length $l$ within a cube of side $L$ or constraining it to have a radius of gyration (which is the root-mean-square distance of the discretized points from their centre of mass) that is less than a pre-assigned value $R$. Even though different boundary conditions influence the optimal string shape, the overall features are found to be robust. Examples of optimal shapes, obtained from numerical simulations, for different ratios of $l / L$ and $l / R$ are shown in Fig. 1 . In both cases, two distinct families of strings, helices and saddles, appear. The two families are close competitors for optimality and different boundary conditions may stabilize one 
over the other. For example, if optimal strings of fixed length are constrained to have a radius of gyration less than $R$, then upon decreasing $R$, the string goes from a regime where the trivial linear string is curled into an arc, then into a portion of helix and finally into a saddle. When the string is constrained to lie within a cube of size $L$, as $L$ decreases first saddles are observed and then helices.

We have also been able to find bulk-like solutions which are not influenced by boundary effects. Such solutions can be obtained by imposing uniform local constraints along the string. On imposing a minimum local density on successive segments of the string (for example, constraining each set of six consecutive beads to have a radius of gyration that is less than a preassigned value $R$ ), we obtained perfectly helical strings, as in Fig. 2, confirming that this is the optimal arrangement. Note that, in close analogy with the sphere-packing problem, the optimal shape displays translational invariance along the chain. In all cases, the geometry of the chosen helix is such that there is an equality of the local radius of curvature (determined by the local bending of the string) and the radius associated with a suitable triplet of non-consecutive points lying in two successive turns of the helix. This is a feature that is observed only for a special ratio $c^{*}$ of the pitch, $p$, and the radius, $r$, of the circle projected by the helix on a plane perpendicular to its axis. When $p / r>c^{*}=2.512$ the global radius of curvature is equal to the local radius with the helix thickness given by $\Delta=r\left(1+p^{2} /(2 \pi r)^{2}\right)$. If $p / r<c^{*}$, the global radius of curvature is strictly lower than the local radius, and the helix thickness is determined basically by the distance between two consecutive helix turns: $\Delta \simeq p / 2$ if $p / r \ll 1$. Optimal packing selects the very special helices corresponding to the transition between the two regimes described above. A visual example is provided by the optimal helix of Fig. 2.

For discrete strings, the critical ratio $p / r$ depends on the discretization level. A more robust quantity is the ratio $f$, averaged over all the points of the string, of the minimum radius of the circles going through each point and any two non-adjacent points and the local radius. For discretized strings, $f=1$ just at the transition described above, whereas $f>1$ in the 'local' regime and $f<1$ in the 'non-local' regime. In our computer-generated optimal strings, $f$ differed from unity by less than a part in a thousand.

It is interesting to note that, in nature, there are many instances of the appearance of helices. For example, many biopolymers such, as proteins and enzymes, have backbones which frequently form helical motifs. (Rose and Seltzer [13] have used the local radii of curvature of the backbone as input in an algorithm for finding the peptide chain turns in a globular protein.) It has been shown [14] that the emergence of such motifs in proteins (unlike in random heteropolymers which, in the melt, have structures conforming to gaussian statistics) is the result of the evolutionary pressure exerted by nature in the selection of native state structures that are able to house sequences of amino acids which fold reproducibly and rapidly 15] and are characterized by a high degree of ther- 
modynamic stability 16. Furthermore, because of the interaction of the amino acids with the solvent, globular proteins attain compact shapes in their folded states.

It is then natural to measure the shape of these helices and assess if they are optimal in the sense described here. The measure of $f$ in $\alpha$-helices found in naturally-occurring proteins yields an average value for $f$ of $1.03 \pm 0.01$, hinting that, despite the complex atomic chemistry associated with the hydrogen bond and the covalent bonds along the backbone, helices in proteins satisfy optimal packing constraints (for the measure of $f$ we considered $\alpha$-helices extracted from the unrelated proteins 1erv, 1 beo and 2end in the Protein Data Bank). This result implies that the backbone sites in protein helices have an associated free volume distributed more uniformly than in any other conformation with the same density. This is consistent with the observation [14] that secondary structures in natural proteins have a much larger configurational entropy than other compact conformations. This uniformity in the free volume distribution seems to be an essential feature because the requirement of a maximum packing of backbone sites by itself does not lead to secondary structure formation [19, 20. Furthermore, the same result also holds for the helices appearing in the collagen native state structure, which have a rather different geometry (in terms of local turn angles, residues per turn and pitch [17]) from average $\alpha$-helices. In spite of these differences, we again obtained an average $f=1.01 \pm 0.03$ (Fig. 3), very close to the optimal situation.

\section{References}

[1] Sloane, N. J. A., Kepler's conjecture confirmed, Nature 395, 435-436 (1998).

[2] Mackenzie, D., Mathematics - proving the perfection of the honeycomb, Science 285, 1339-1340 (1999).

[3] Woodcock, L. V., Entropy difference between the face-centered cubic and the hexagonal close-packed crystal structures, Nature 385, 141-143 (1997).

[4] Car, R., Crystal structure - how hard spheres stack up, Nature 385, 115116 (1997).

[5] Cipra, B., Mathematics - packing challenge mastered at last, Science 281, 1267 (1998).

[6] Stewart, I., Tight tins for round sardines, Sci. Am. 278, 80-82, Feb. 1998.

[7] Buck, G. and Orloff, J., A simple energy function for knots, Topol. Appl. 61, 205-214 (1995).

[8] Katritch, V., Bednar, J., Michoud, D., Scharein, R. G., Dubochet, J. and Stasiak, A., Geometry and physics of knots, Nature 384, 142-145 (1996). 
[9] Katritch, V., Olson, W. K., Pieranski, P., Dubochet, J. and Stasiak, A., Properties of ideal composite knots, Nature 388, 148-151 (1997).

[10] Gonzalez, O. and Maddocks, J. H., Global curvature, thickness and the ideal shapes of knots, Proc. Natl. Acad. Sci. USA 96, 4769-4773 (1999).

[11] Buck, G., Four thirds power law for knots and links, Nature 392, 238-239 (1998).

[12] Cantarella, J., Kusner, R. B. and Sullivan, J. M., Tight knot values deviate from linear relations, Nature 392, 237-238 (1998).

[13] Rose, G. D. and Seltzer, J. P., A new algorithm for finding the peptide chain turns in a globular protein, J. Mol. Biol. 113, 153-164 (1977).

[14] Micheletti, C., Maritan A., Banavar, J. R. and Seno, F., Protein structures and optimal folding from a geometrical variational principle. Phys. Rev. Lett. 82, 3372-3375 (1999).

[15] Maritan, A., Micheletti, C. and Banavar, J. R., Role of secondary motifs in fast folding polymers: a dynamical variational principle, Phys. Rev. Lett. 84, 3009-3012 (2000).

[16] Sali, A., Shakhnovich, E. and Karplus, M., How does a protein fold, Nature 369, 248-251 (1994).

[17] Creighton, T. E., Proteins - Structures and Molecular Properties, W. H. Freeman and Company, New York (1993), pag. 182-188.

[18] Sokal, A. D., Monte Carlo methods for the self-avoiding walk, Nuclear Physics B47, 172-179 (1996).

[19] Yee, D. P., Chan, H. S., Havel, T. F. and Dill, K. A., Does compactness induce secondary structure in proteins? Study of poly-alanine chains computed by distance geometry, J. Mol. Biol. 241, 557-573 (1994).

[20] Socci, N. D., Bialek, W. S. and Onuchic J. N., Properties and origins of protein secondary structure, Phis. Rev. E 49, 3440-3443 (1994).

Acknowledgements This work was supported by INFN, NASA and The Donors of the Petroleum Research Fund administered by the American Chemical Society. A.T. thanks the Physics Department of Università degli Studi di Padova, Padova, Italy, for its hospitality. 


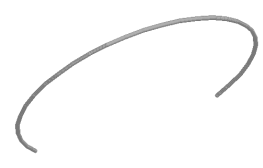

(a)

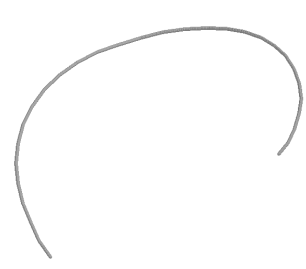

(d)

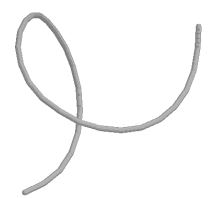

(b)

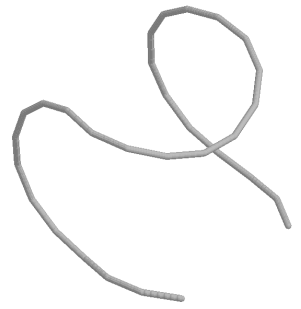

(e)

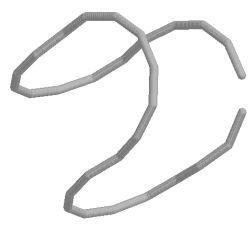

(c)

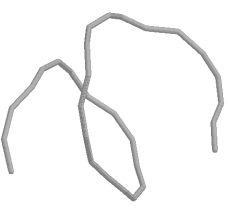

(f)

Figure 1: Examples of optimal strings. The strings in the figure were obtained starting from a random conformation of a chain made up of $N$ equally spaced points (the spacing between neighboring points is defined to be 1 unit) and successively distorting the chain with pivot, crankshaft and slithering moves commonly used in stochastic chain dynamics 18. A Metropolis Monte Carlo procedure is employed with a thermal weight, $e^{+\Delta / T}$, where $\Delta$ is the thickness and $T$ is a fictitious temperature set initially to a high value such that the acceptance rate is close to 1 and then decreased gradually to zero in several thousand steps. Self-avoidance of the optimal string is a natural consequence of the maximization of the thickness. The introduction of a hard-core repulsion between beads was found to significantly speed up convergence to the optimal solution and avoid trapping in self-intersecting structures. We have verified that the same values (within 1 percent) of the final thickness of the optimal strings are obtained starting from unrelated initial conformations. Top row: optimal shapes obtained by constraining strings of 30 points with a radius of gyration less than $R$. (a) $R=6.0, \Delta=6.42$ (b) $R=4.5, \Delta=3.82$ (c) $R=3.0, \Delta=1.93$. Bottom row: optimal shapes obtained by confining a string of 30 points within a cube of side $L$. (d) $L=22.0, \Delta=6.11$ (e) $L=9.5, \Delta=2.3$ (f) $L=8.1$, $\Delta=1.75$. 


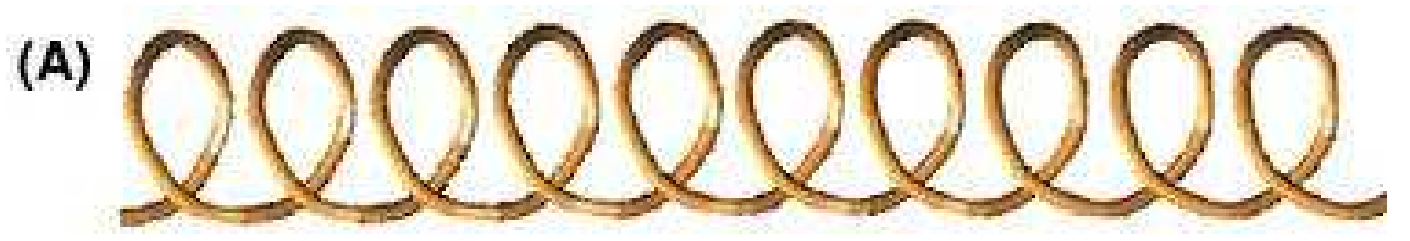

(B)

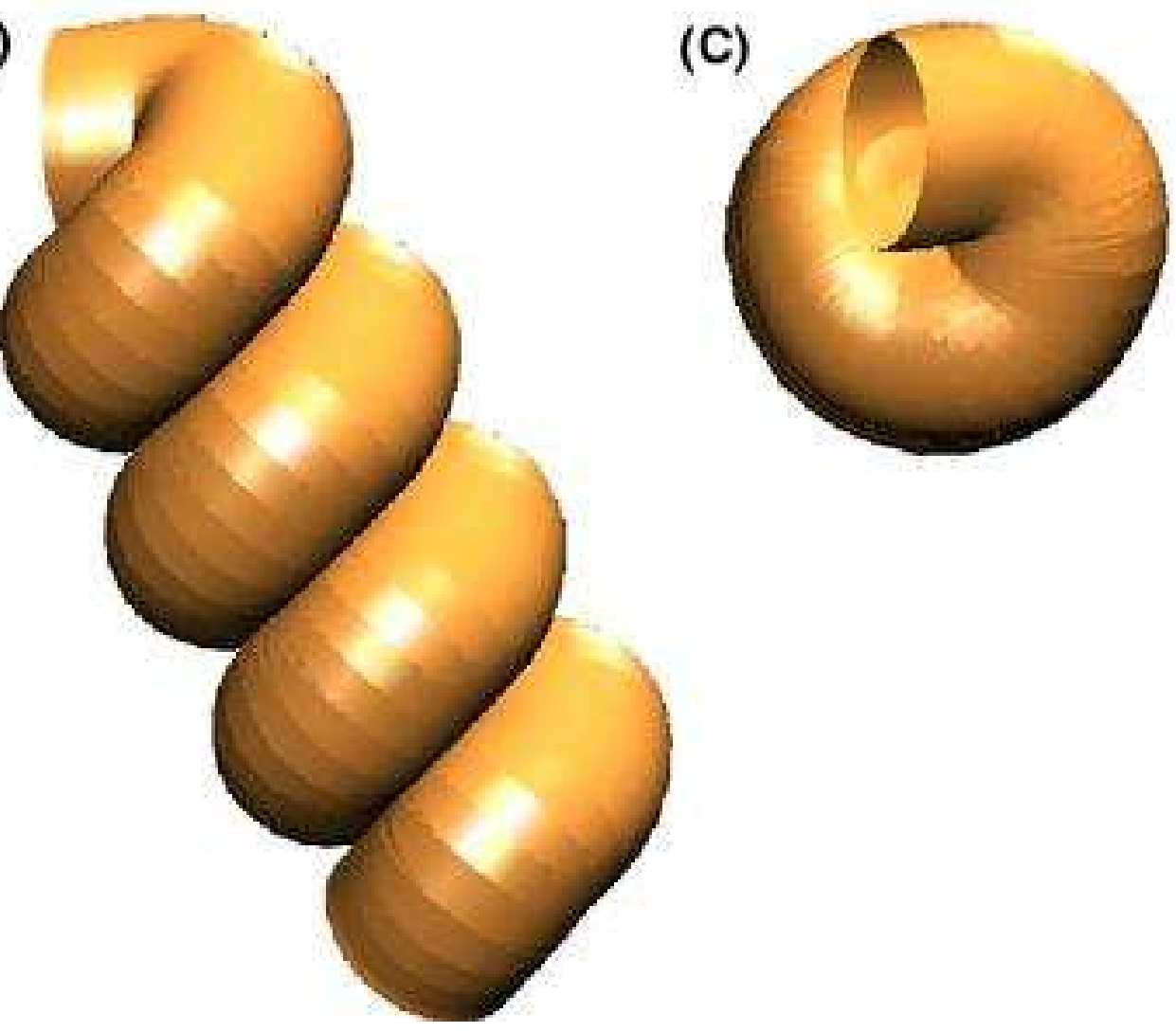

Figure 2: Optimal string with local constraint. The string has 65 points with a neighbor separation of unity. The local constraint was that each set of six consecutive beads had a radius of gyration less than 1 . The value of $f$ (see text) for this string is 0.9993 . This result is quantitatively the same for a broad class of local constraints. Panel A shows the "bare" skeleton of the optimal helix connecting the discrete beads. Panels B and $\mathrm{C}$ present side and top views of the same helix inflated to its thickness. Note that there is no free space either between consecutive turns of the helix or in the plane perpendicular to the helix axis. 


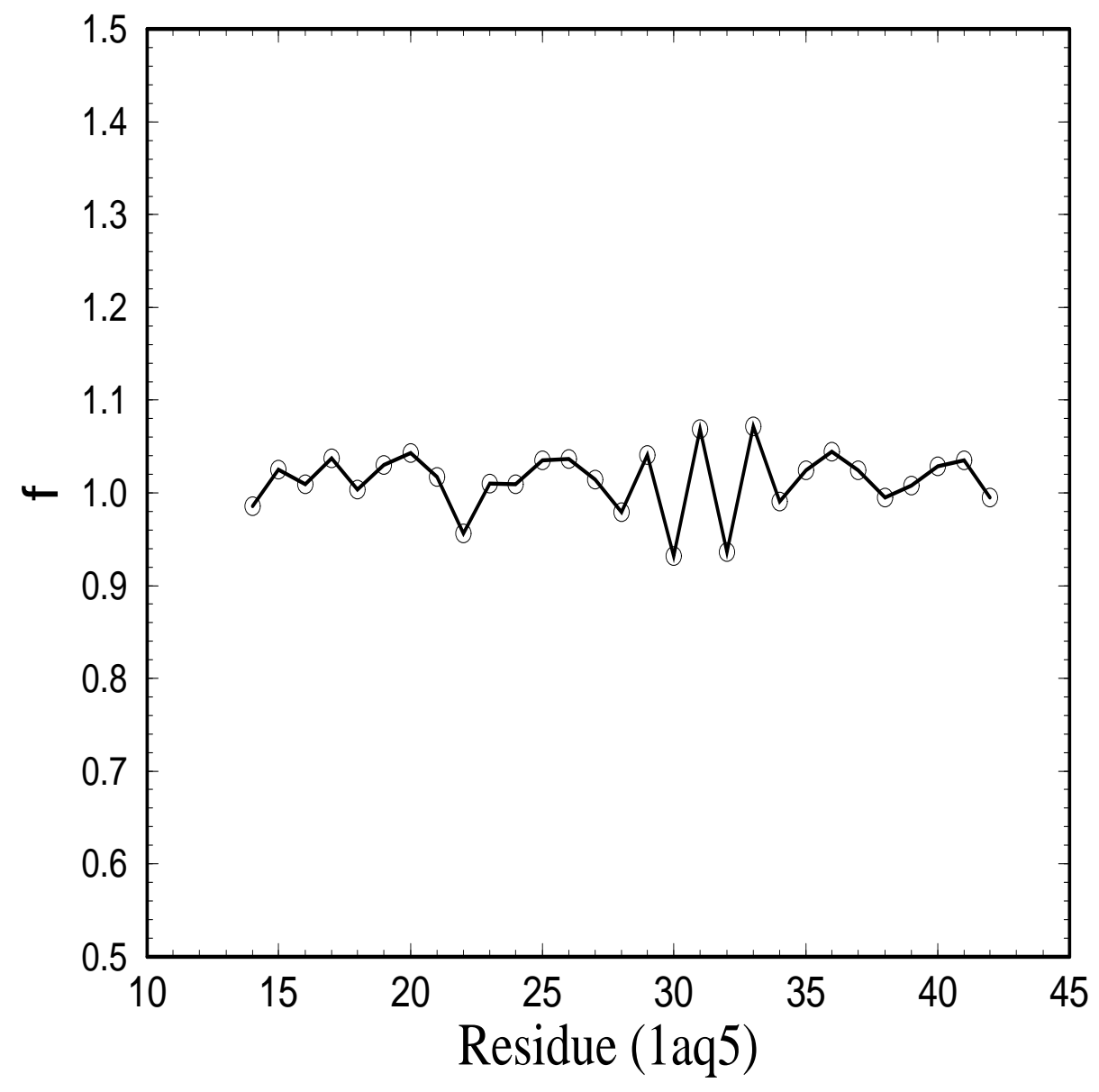

Figure 3: Packing of collagen helices. Plot of $f$ values as a function of sequence position for a single collagen helix (only $C_{\alpha}$ coordinates were used to identify the protein backbone). The same plot for each of the three collagen chains would simply superimpose. We considered the residues 14-42 from the structure 1aq5 in the Protein Data Bank. 